\title{
MODELLING THE HELICOPTER ROTOR AERODYNAMICS AT FORWARD FLIGHT WITH FREE WAKE MODEL AND URANS METHOD
}

\author{
Yuri IGNATKIN (D) *, Pavel MAKEEV (D), Sergey KONSTANTINOV (D), Alexander SHOMOV (D) \\ Helicopter Design Department, Moscow Aviation Institute (National Research University), \\ Moscow, Russian Federation
}

Received 30 January 2020; accepted 8 May 2020

\begin{abstract}
The presented work is dedicated to the numerical study of the aerodynamic characteristics of the helicopter rotor. Two approaches to modeling of the rotor are applied: the free wake model developed by the Authors with using steady airfoil characteristics and the Unsteady RANS method based on the Ansys Fluent software. The modes of hovering and horizontal flight in the range of advancing ratio $\mu=(0-0.45)$ are considered. The shapes of the rotor wake, the distributions of the normal force coefficient and the fields of inductive velocities for all considered flight modes are calculated. For a particular case with $\mu=0.25$ there is a comparison with experimental data. The time needed for calculation of the applied methods is estimated. Accuracy of the used methods in the framework of the solved task is analysed with taking into account used models assumptions. It is shown that in the range of $\mu=(0-0.25)$ the free wake model provides a fast and reliable calculation of the aerodynamic characteristics of the helicopter rotor. For values of $\mu>0.35$ it is necessary to take into account the unsteady characteristics of the airfoil.
\end{abstract}

Keywords: helicopter rotor, free wake model, URANS method, forward flight, aerodynamic characteristics.

\section{Introduction}

The aerodynamic characteristics of the helicopter rotor in the forward flight significantly depend on the vortical wake created by the rotor blades. The rotor wake at low and medium flight speeds is characterised by significant nonlinearity and complexity of its structure (Leishman, 2006; Johnson, 2013). The vortices interact with the rotor blades (BVI - phenomenon), having a significant impact on distribution of aerodynamic loads along the blade. In the rotor wake the interaction of vortices leads to the rolling up of the wake and the formation of two powerful rotor disc vortices.

Therefore, to solve practical tasks and problems related to determination of the aerodynamic characteristics of the rotor, it is necessary to take into account the deformation of the rotor wake under influence of its inductive velocity field.

Currently, FVM are widely used to solve practical tasks of helicopter rotor aerodynamics. It is generally accepted to distinguish two large classes of vortex models. The first type includes grid models based on VLM, where the free wake is modeled by the grid of vortex segments (Bhagwat \& Leishman, 2000; Long \& Fritz, 2004; Shcheglova, 2011;
Colmenares et al., 2015; Dominique et al., 2018). The second type is based on VPM (He \& Zhao, 2009; He \& Rajmohan, 2016; Singh \& Friedmann, 2017; Tan et al., 2018; Alvarez \& Ning, 2018). The blade of the rotor can be modeled using the lifting line theory (Shcheglova, 2011) or the lifting surface theory. Also the hypothesis of plane sections is used (Leishman, 2006). A feature of the vortex models is necessity to use external data on the aerodynamic characteristics of the airfoils. These data are usually based on the wind tunnel test results or computational methods.

With the growth of computational capabilities, methods of computational fluid dynamics, based on the solution of the Navier-Stokes equations by iterative method using the finite volume method, are also widely used. The most common is to use the RANS / Unsteady RANS (Reynolds-Averaged-Navier-Stokes) method as the least resource-intensive (Wenping et al., 2007; Potsdam et al., 2009; Garipova et al., 2015; Kelly et al., 2015; Deng et al., 2019; Kinzel et al., 2019). It is also possible to use the DES (Detached-Eddy Simulation) method. DES allows clarifying the results obtained in comparison with the URANS method, especially the question of BVI, but it is extremely demanding for computing resources (Dehaeze et al., 2018). CFD methods make it possible to simulate

*Corresponding author. E-mail: ignatkinym@mai.ru

Copyright (C) 2020 The Author(s). Published by Vilnius Gediminas Technical University.

This is an Open Access article distributed under the terms of the Creative Commons Attribution License (https://creativecommons.org/licenses/by/4.0/), which permits unrestricted use, distribution, and reproduction in any medium, provided the original author and source are credited. 
the dynamics of fluid flows taking into account the effects of viscosity, compressibility and stall phenomenon. These methods have a wide information content and potentially higher accuracy than vortex methods, but they are very sensitive to the choice of the turbulence model and the quality of the meshes (Ignatkin \& Konstantinov, 2012). The disadvantage of such methods is extremely large computational resources necessary to solve tasks related to the helicopter rotor aerodynamics (Kritsky et al., 2016).

Coupled methods combining the vortex theory for free wake modeling and CFD methods for modeling the flow around the blade are also widely used (Bhagwat et al., 2006; Farrokhfal \& Pishevar, 2014; Zhao et al., 2017; Shi et al., 2017). This approach allows combining high performance calculations with high accuracy.

The presented work is concentrated on comparative analysis of the capabilities of two modern computational methods on the example of numerical simulation of helicopter rotor aerodynamic characteristics at the forward flight.

\section{Object and methods of study}

Parameters of the rotor and calculation. Calculation of aerodynamic characteristics was performed for the testing model of a rotor with the following parameters: number of blades is $N_{\mathrm{b}}=4$; rotor solidity $\sigma=0.133$; linear speed of the blade tips $\omega R=45.24 \mathrm{~m} / \mathrm{s}$; the blade pitch angle $\theta=8^{\circ}$; the twist of the blade $\theta_{t w}=0^{\circ}$; blade chord $c=0.15 \mathrm{~m}$; the radius of the rotor $R=1.2 \mathrm{~m}$; the radius of the blade root section $r_{0}=0.2 \mathrm{~m}$, angle of attack of the rotor $\alpha_{R}=0^{\circ}$. The rotor is absolutely rigid and does not make the flapping motions relative to the hinges. The blades are rectangular in plan with a profile NACA 0012.

Calculations of the aerodynamic characteristics of the rotor on the hover mode and on the forward flight with advance ratio $\mu=0.05 ; 0.1 ; 0.15 ; 0.25 ; 0.35 ; 0.45$ have been performed.

Free wake model. The basis of the model developed at the Department of "Helicopter Design" MAI is the lifting line theory (Ignatkin et al., 2009). The rotor blade is modeled by an attached vortex located on the quarter chord of the blade. To calculate the aerodynamic characteristics of the blade, the hypothesis of plane sections is used. The steady aerodynamic characteristics of the airfoils obtained by the wind tunnel tests at the real values of the Reynolds and Mach numbers are used. The blade is absolutely rigid, as in most vortex models for calculating the aerodynamic characteristics of the rotor (Bhagwat \& Leishman, 2000). The model allows considering the flapping motions of the blades relative to the horizontal hinge (Long \& Fritz, 2004).

The vortex lattice method used the system of longitudinal and a transverse vortex departs from each rotor blade. Thus the free vortex wake behind the blade is a grid of quadrangular elements (Colmenares et al., 2015). Each element consists of vortex segments. The free vortex wake is deformed over time under the influence of the velocity of the free stream, as well as induced velocities from the vortex wake. As a result of the calculation, a free vortex wake is built step by step behind the rotor.

A feature of the vortex system used in the computational model is the representation of vortex segments as diffusing vortex lines (Shcheglova, 2011). This makes possible to simulate physical processes, occurring in the rotor wake, more accurately. Thus, it is possible to avoid mathematical features that lead to rapid destruction of the grid and the inability to simulate a lot of flight modes (Homayoun \& Amir, 2003).

The used free wake model is characterised by low demands on computing resources (Kritsky et al., 2016). The calculations based on this model were performed on a personal computer with CPU Intel Core i7 equipped with 6 computational cores (12 threads). To provide highresolution wake shapes of the rotor azimuthal step was $\Delta \psi=3^{\circ}$. The calculation of one mode took about 20 hours for 10 revolutions of the rotor.

The computational model of the rotor is shown on Figure 1.

URANS method. Numerical simulation of the rotor by the URANS method was carried out in unsteady setting using a mesh with a sliding boundary interface. The calculations were performed on the supercomputer with using the ANSYS FLUENT software. The RANS closed by the Spalart-Allmaras turbulence model were used to describe the motion of a viscous turbulent flow of a compressible gas (Dindar et al., 2000; Ignatkin \& Konstantinov, 2012).

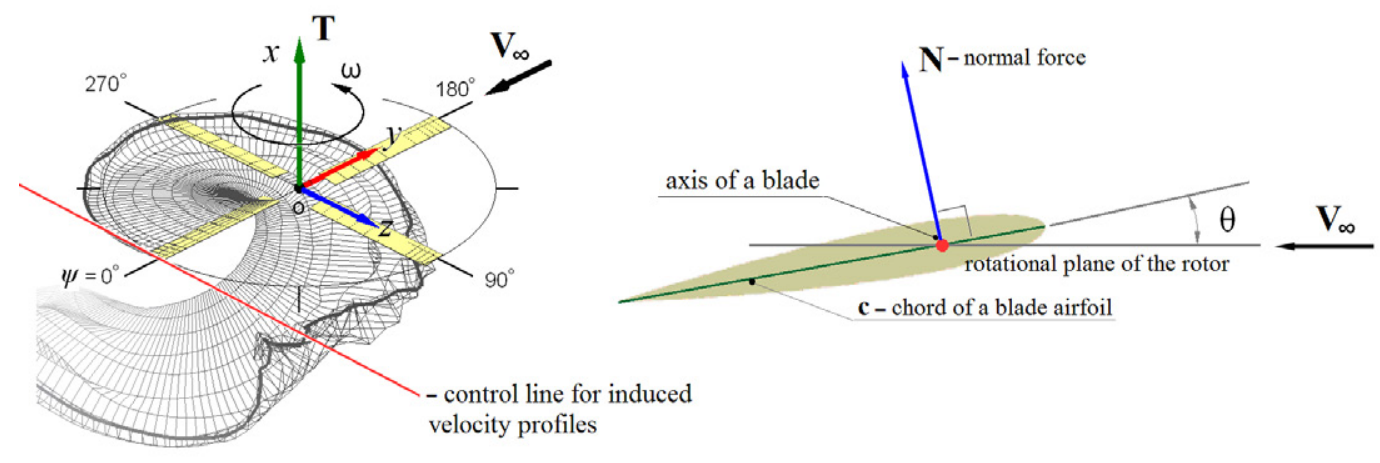

Figure 1. Computational model of a rotor based on free wake model 

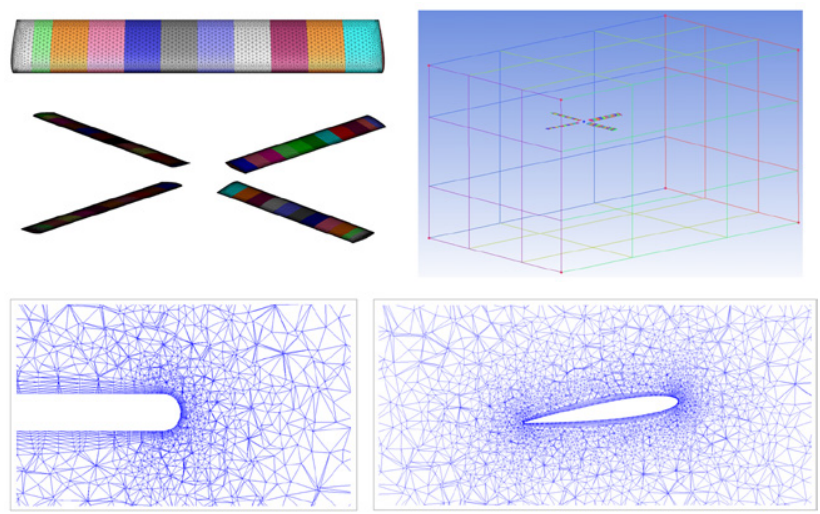

Figure 2. The model of the blade/rotor and the computational mesh used of the URANS method

The calculated domain was a rectangular shape (Figure 2). The lateral boundaries of the computational domain were located from the axis of the model at a distance of $2.5 R$, the upper at a distance of $1.5 R$, the lower and input boundaries at a distance of $3 R$, the output boundary at a distance of $5 R$. The blades were divided along the radius into 10 equal sections, the blade end section was additionally divided into two equal parts.

The calculated mesh had an unstructured topology and was built automatically. The mesh contains about fifteen million cells of tetrahedra and prisms. To determine reliably the aerodynamic characteristics of the rotor, the calculated mesh was adapted to the characteristics of the flow around the blades and was built with an increase of cells number in the end and end sections of the blades (Figure 2). To take account surface flow, a prismatic layer consisting of twelve prismatic cells, was created on the surface of the blade. The prismatic layer was constructed in such a way that the first node of the prismatic cell was located in a viscous sublayer $\left(Y^{+} \leq 1\right)$. The initial turbulence parameters were based on the average intensity of the mature turbulent flow. The value of the relative turbulent viscosity was assumed to be equal to 3 .

Calculations based on the RANS method were carried out with using of supercomputer with 150 cores. The calculation of one mode took about 160 hours for 10 revolutions of the rotor with azimuthal step $\Delta \psi=0.216^{\circ}$

\section{Presentation of the results and discussion}

As a result of the calculations, the pictures of visualisation of the rotor wake shapes, diagrams of the vertical component of the inductive velocities $v_{y}$ in the wake of the rotor, as well as diagrams of the distribution of the normal (normal to blade chord) force coefficient $C_{n}$ (Leishman, 2006) along the radius of the blade at various azimuths were obtained.

At the first stage of the study, the vortex wake shapes and the dependence of $C_{\mathrm{T}}=\mathrm{f}\left(C_{\mathrm{Q}}\right)$ (power polar) of the rotor in the hover were calculated. Figure 3 and Figure 4 presents these results by both used methods. In Figure 3

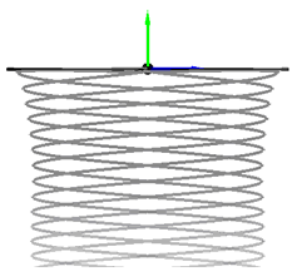

a)

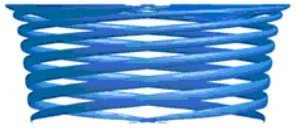

b)
Figure 3. Comparison of rotor wake visualisation at hover by URANS method (a) and by free-wake model (b)

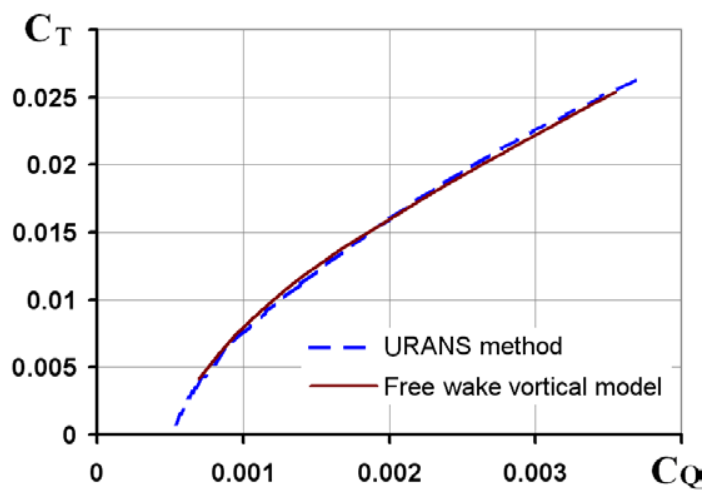

Figure 4. Calculated dependences of $C T=f(C Q)$ in hover

there are results of visualisation of the free vortex wake obtained by free wake model (in this case a tip vortex trajectories have been visualised) and by URANS method (using isosurface). The presented results show good coincidence of rotor wake shapes. The power polar presented in the Figure 4 also shows good coincidence of rotor thrust and torque coefficient in hovering mode (Garipova et al., 2015) obtained by both methods.

The results of visualisation of the rotor wake shapes at the rotor advancing ratio $\mu=(0.05-0.45)$ are shown in Figure 5. Starting with advancing ratio value of $\mu=0.05$ (see Figure 5a) the formation of vortex wake into the system of the right and left rotor disc vortexes is observed (Shi et al., 2017; Tan et al., 2018). This feature of the vortex system is mostly characteristic for $\mu=(0.1-0.25)$ (see Figure $5 b-d$ ). In fact, for all considered modes, the results of visualization of the rotor wake shapes on the basis of the free wake model and the RANS method are in good agreement with each other.

Figures 6 shows calculated distributions of the coefficients of the normal force $C_{\mathrm{n}}$ along the radius of the blade for different azimuthally position for the advancing ratio values $\mu=(0-0.45)$. There is a satisfactory agreement between the results of calculations based on the free wake model and the RANS method for the values $\mu=(0.05$ 0.25 ) (Figures $6 \mathrm{a}-\mathrm{d}$ ). For $\mu=0.25$, the results of an experiment in a wind tunnel (Belotserkovskii \& Loktev, 1992) are additionally presented, confirming the reliability of the obtained calculations results (Figure 6d). At the $\mu=0.35$ (Figure 6e) and $\mu=0.45$ (Figure 6f), there are observed significantly differences between the results of normal 


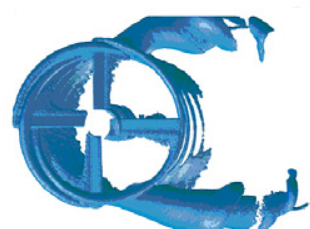

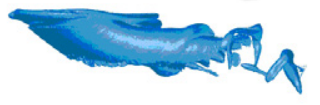

URANS method
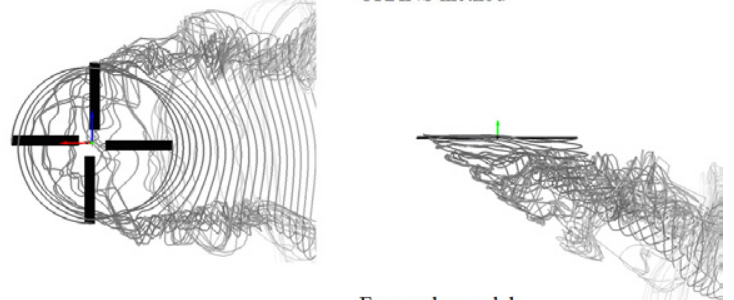

Free wake model

a) $\mu=0.05$
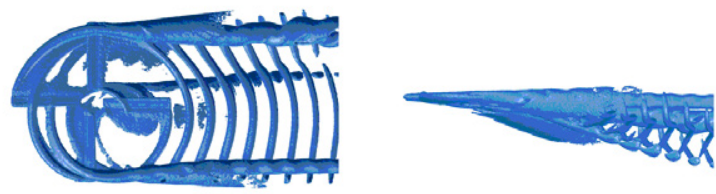

URANS method
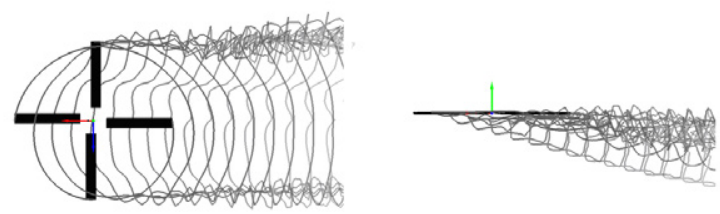

Free wake model

c) $\mu=0.15$
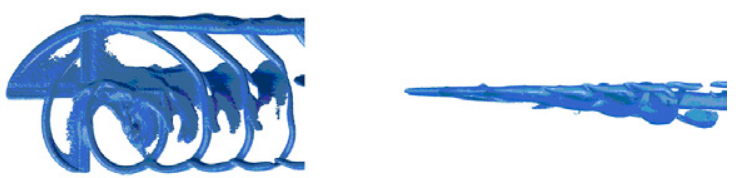

URANS method

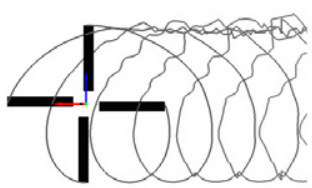

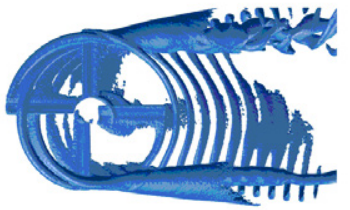

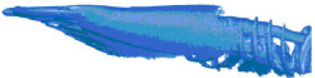

URANS method
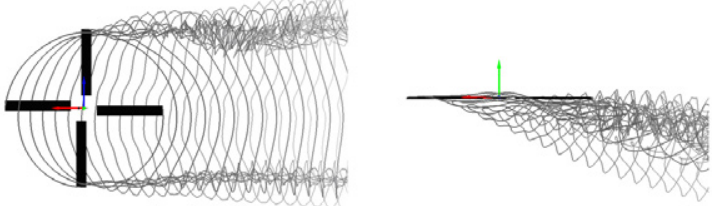

Free wake model

b) $\mu=0.1$
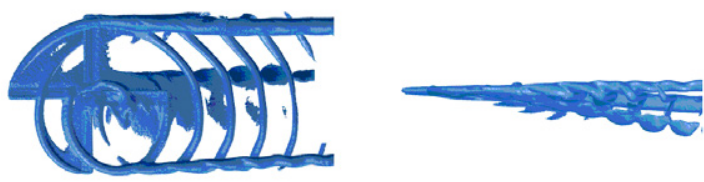

URANS method
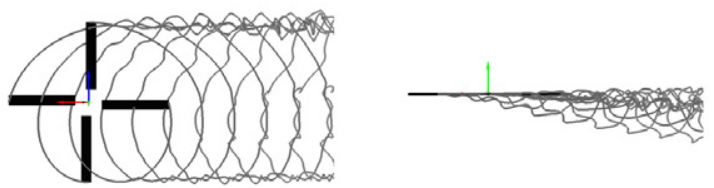

Free wake model

d) $\mu=0.25$

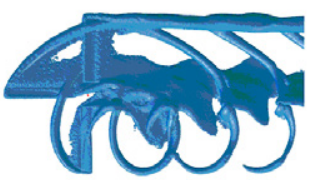

URANS method
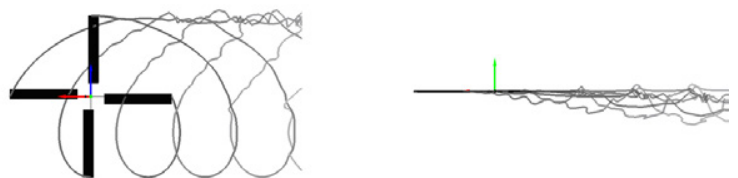

Free wake model

f) $\mu=0.45$

Figure 5. Comparison of rotor wake visualisation at the forward flight received by URANS method (isosurface) and by free wake model (blade tip vortex trajectories) 

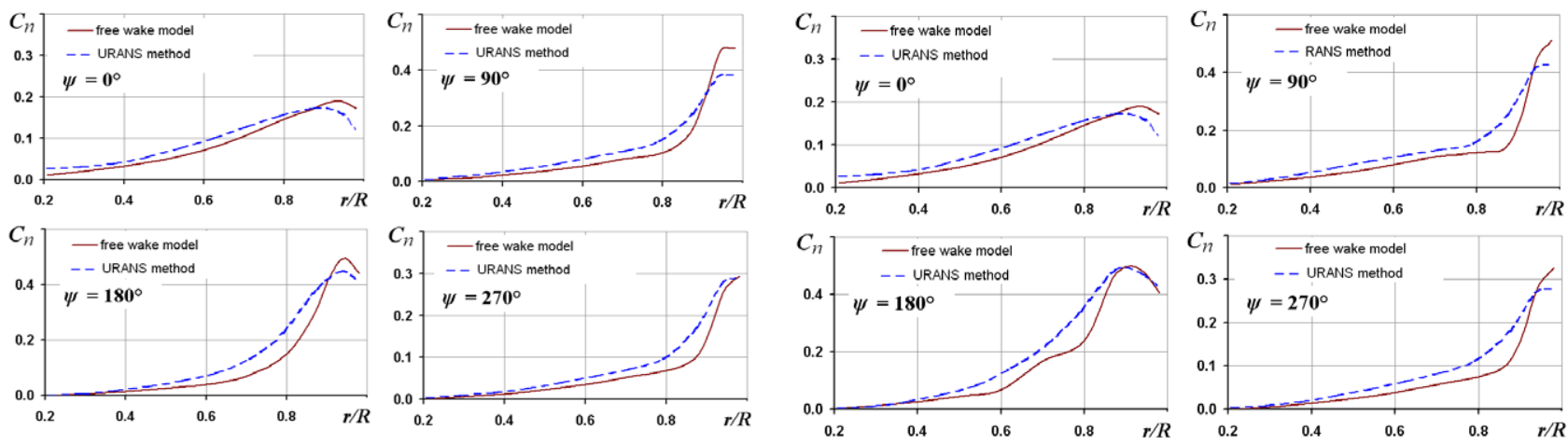

a) $\mu=0.05$
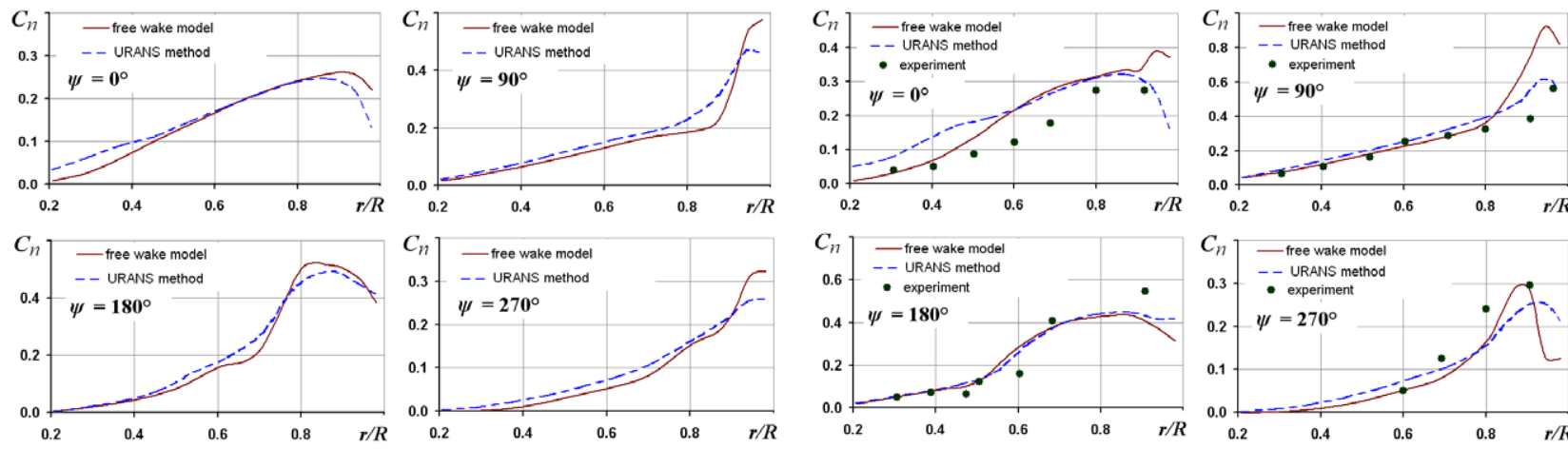

c) $\mu=0.15$
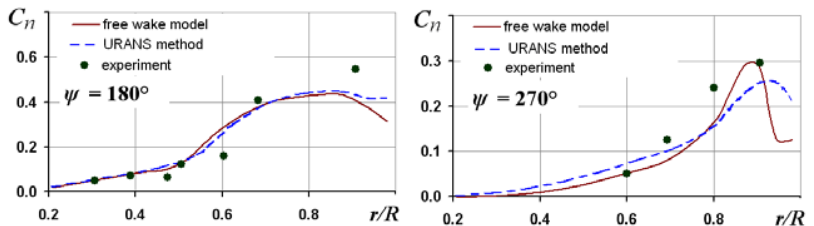

d) $\mu=0.25$
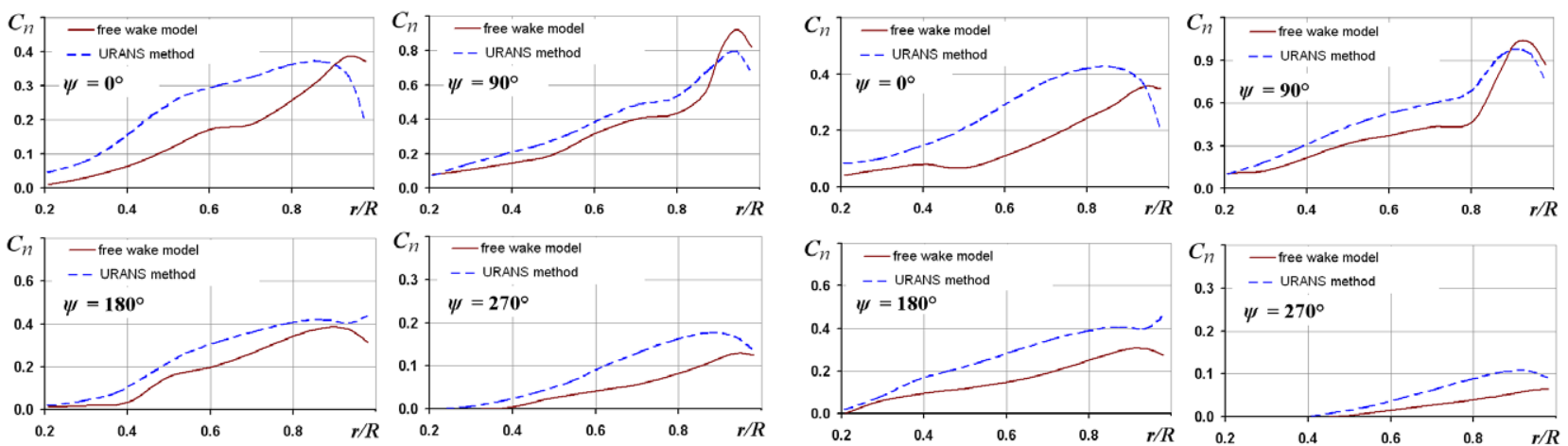

e) $\mu=0.35$
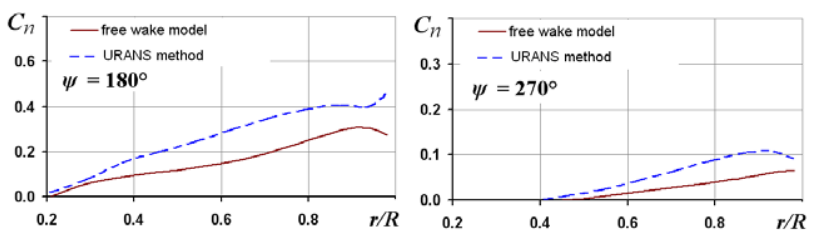

f) $\mu=0.45$

Figure 6. Comparison of normal force coefficient distribution $C_{n}=\mathrm{f}(r / R)$ at various blade azimuthally location by URANS method and free wake model

force coefficient distributions obtained by both methods. Meanwhile, with growth $\mu$ values from 0.35 to 0.45 the difference of obtained results is also growing up. This fact is obviously related to the assumptions of the free wake model using the steady airfoil characteristics and the plane section hypothesis (Leishman, 2006; Johnson, 2013).

Figure 7 shows the calculated diagrams of inductive velocity profiles in the wake of the rotor on the studied modes. Diagrams of inductive velocities were determined along the control line located in the coordinate system associated with the rotor OXYZ (see Figure 1). The diagrams show a vertical projection of the inductive velocity. The diagrams of inductive velocities obtained on the basis of calculations by the free wake model and URANS method satisfactorily coincides at the values of $\mu=(0-0.35)$ (Figure $7 \mathrm{~b}-\mathrm{e})$. Differences are observed at $\mu=0.05$ (Figure 7a) and $\mu=0.45$ (Figure 7f). In the first case $(\mu=0.05)$, the differences of the inductive velocity diagrams can be explained by the high complexity of the vortex wake shape at low advancing ratio value. In the second case $(\mu=0.45)$, the cause may be identified with significant differences in aerodynamic characteristics obtained on the basis of the methods used (see Figure 6). This is due to the assumptions made in the used free wake model, first of all, using stationary aerodynamic characteristics of airfoils. 


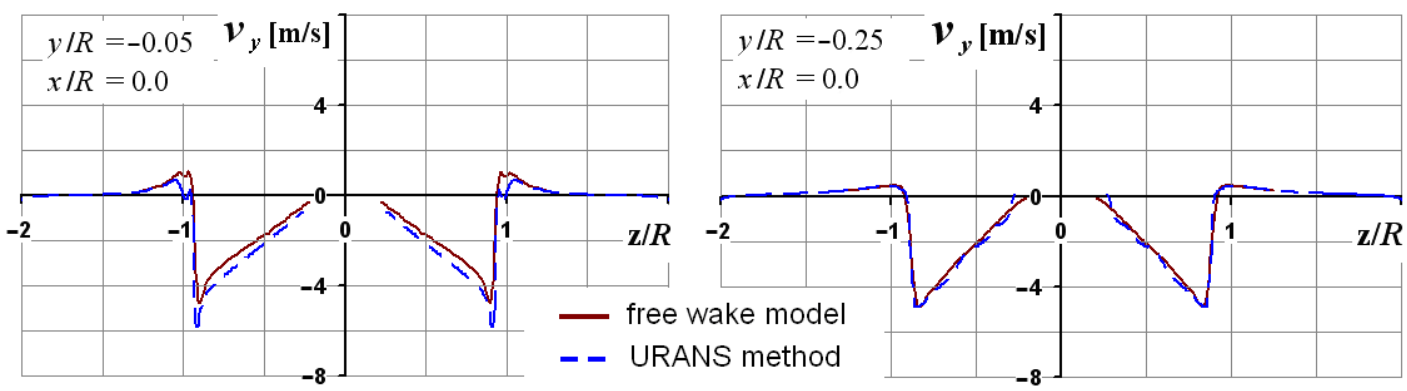

$\mu=0$ (for $y / R=-0.05 ;-0.25$ and $x / R=0.0$ )

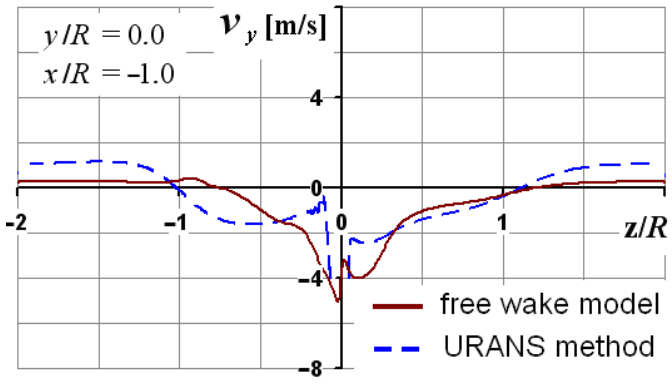

$\mu=0.05$

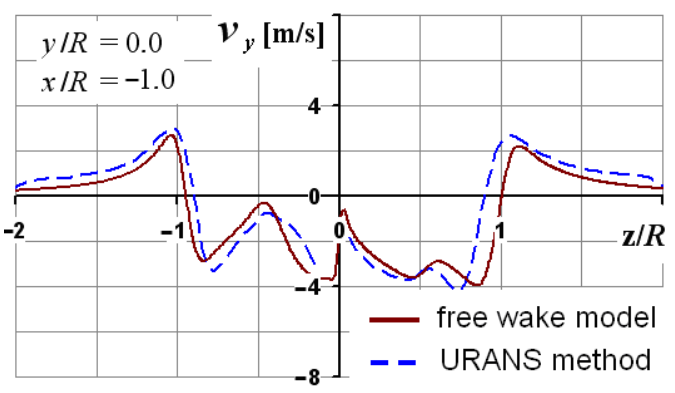

$\mu=0.15$

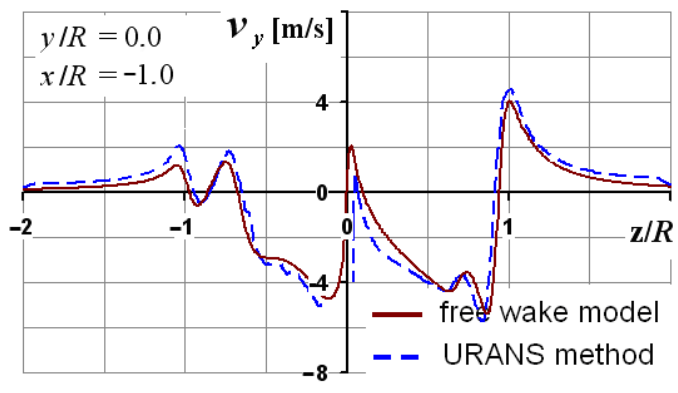

$\mu=0.35$

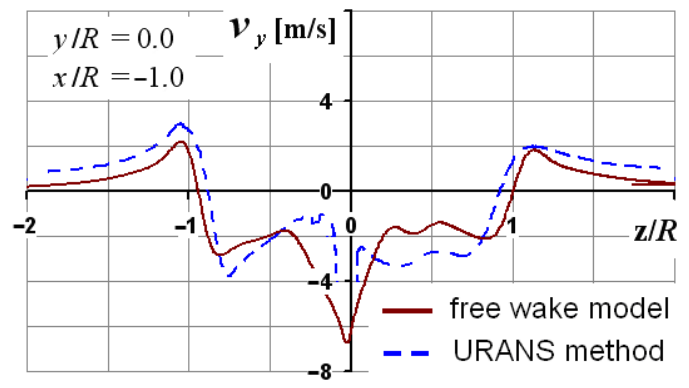

$\mu=0.1$

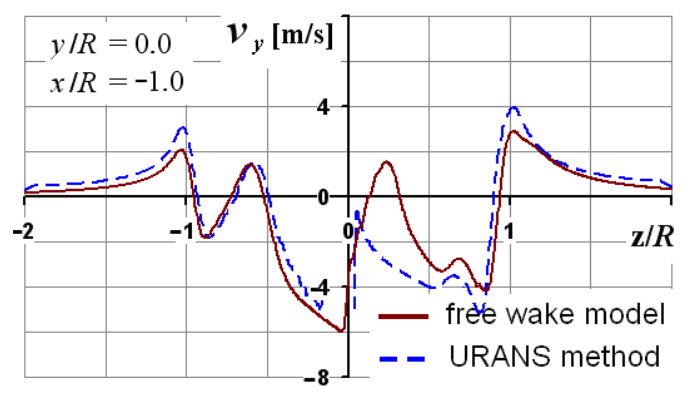

$\mu=0.25$

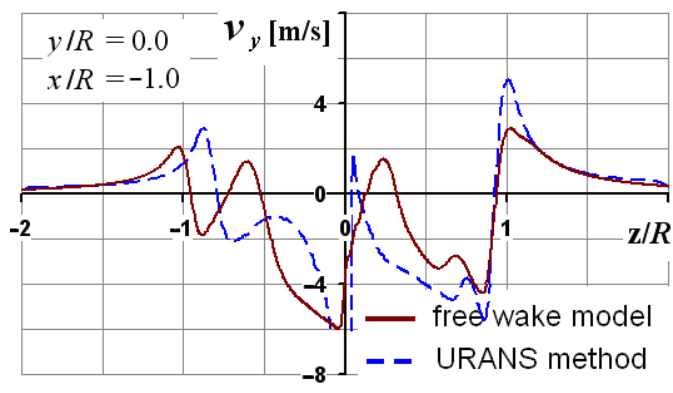

$\mu=0.45$

Figure 7. Comparison of inductive velocity profile $v_{y}=\mathrm{f}(z / R)$ for $y / R=0$ and $x / R=-1.0$ by URANS method and free wake model

\section{Conclusions}

On the basis of created by the Authors free vortex wake model and the URANS method (based on Ansys Fluent software), a comparative numerical study of the helicopter main rotor aerodynamic at the forward flight with advance ratio values of $\mu=(0-0.45)$ is performed.

New results of visualisation of the vortex wake shapes are obtained. These results illustrate the characteristic fea- tures of vortex wake structure for helicopter rotor in forward flight at different advance ratio values.

The vortex wake shapes and induced velocities fields data obtained by both used methods match the whole range of advance ratio values under consideration.

The analysis of blade distribution of the normal force coefficient for various azimuth angles shows that in the range of $\mu=(0.05-0.25)$ the results of both methods match 
satisfactorily. For large values of $\mu>0.25$, there are significant differences in the calculated aerodynamic loads.

The results of comparative study confirm the proposed limits in use of the vortex model for modeling high values of forward flight speeds $(\mu>0.25)$. These limits are related to the application of the lifting line theory with plane section hypothesis and usage of steady airfoil characteristics. The URANS method is free of these assumptions.

Therefore in the speed range of $\mu=0 . .0 .25$, both approaches give similar results, when calculating total and distributed characteristics of the rotor forms a vortex wake and fields of induced velocities.

The estimation of the required calculation time showed a significant calculating speed of the created free wake model in comparison with the URANS methods. It is shown that the free wake model does not require special supercomputer clusters for the same calculation performing.

The obtained results confirm the prospects of using the free wake model for solving a wide range of practical tasks of helicopter rotor aerodynamics, especially at the low and medium flight speeds. This includes the case of calculating multi-rotor configurations that are still difficult for modeling, based on the URANS method and similar high-order approaches.

To expand developed free wake model unsteady aerodynamic characteristics of the blade airfoils should be implemented in the model. In the future, this may allow using a developed vortex model when modeling the helicopter rotor aerodynamics at forward flight speeds of $\mu>0.25$.

\section{References}

Alvarez, E. J., \& Ning, A. (2018). Development of a vortex particle code for the modeling of wake interaction in distributed propulsion. Proceedings of AIAA Applied Aerodynamics Conference (p. 22). Atlanta, USA. https://doi.org/10.2514/6.2018-3646

Belotserkovskii, S. M., \& Loktev, B. E. (1992). Computer-aided study of aerodynamic and aeroelastic characteristics of the helicopter rotors. Mashinostroenie.

Bhagwat, M. J., \& Leishman, J. G. (2000). Time-accurate modeling of rotor wakes using a free-vortex wake method. Proceedings of 18th AIAA Applied Aerodynamics Conference (pp. 236-246). Denver, USA. https://doi.org/10.2514/6.2000-4120

Bhagwat, M., Moulton, M. A., \& Caradonna, F. X. (2006). Hybrid CFD for rotor hover performance prediction. Proceedings of 24th Applied Aerodynamics Conference. San Francisco, USA. https://doi.org/10.2514/6.2006-3474

Colmenares, J. D., Lopez, O. D., \& Preidikman, S. (2015). Computational study of a transverse rotor aircraft in hover using the unsteady vortex lattice method. Mathematical Problems in Engineering, 1, 1-9. https://doi.org/10.1155/2015/478457

Dehaeze, F., Barakos, G. N., Kusyumov, A. N., Kusyumov, S. A., \& Mikhailov, S. A. (2018). Exploring the detached-eddy simulation for main rotor flows. Russian Aeronautics, 61(1), 37-44. https://doi.org/10.3103/S1068799818010063

Deng, J., Fan, F., Liu, P., Huang, S., \& Lin, Y. (2019). Aerodynamic characteristics of rigid coaxial rotor by wind tunnel test and numerical calculation. Chinese Journal of Aeronaut, 32(3), 568-576. https://doi.org/10.1016/j.cja.2018.12.026
Dindar, M., Shephard, M. S., Flaherty, J. E., \& Jansen, K. (2000). Adaptive CFD analysis for rotorcraft aerodynamics. Computer Methods in Applied Mechanics and Engineering, 189, 10551076. https://doi.org/10.1016/S0045-7825(99)00368-0

Dominique, F., Lone, M., Weber, S., \& Sharma, A. (2018). Fast computational aeroelastic analysis of helicopter rotor blades. Proceedings of AIAA Aerospace Sciences Meeting, 1-23.

Farrokhfal, H., \& Pishevar, A. R. (2014). A new coupled free Wake-CFD method for calculation of helicopter rotor flowfield in hover. Journal of Aerospace Technology and Management, 6(2), 129-147. https://doi.org/10.5028/jatm.v6i2.366

Garipova, L. I., Batrakov, A. S., Kusyumov, A. N., Mikhailov, S. A., \& Barakos, G. N. (2015). Estimates of hover aerodynamics performance of rotor model. Russian Aeronautics, 57(3), 223231. https://doi.org/10.3103/S1068799814030027

He, C., \& Rajmohan, N. (2016). Modeling the aerodynamic interaction of multiple rotor vehicles and compound rotorcraft with viscous vortex particle method. Proceedings of AHS 72nd Annual Forum, 18.

He, C., \& Zhao, J. (2009). Modeling rotor wake dynamics with viscous vortex particle method. AIAA Journal, 47(4), 902915. https://doi.org/10.2514/1.36466

Homayoun, E., \& Amir, N. (2003). Application of vortex lattice and quasi-vortex lattice method with free wake in calculation of aerodynamic characteristics of a hovering helicopter rotor blade in ground effect. Scientia Iranica, 10(1), 84-90.

Ignatkin, Y. M., \& Konstantinov, S. G. (2012). Researches of aerodynamic characteristics of a main rotor helicopter using CFD method. Trudy MAI, 57, 1-22.

Ignatkin, Y. M., Makeev, P. V., Grevtsov, B. S., \& Shomov, A. I. (2009). A nonlinear blade vortex propeller theory and its applications to estimate aerodynamic characteristics for helicopter main rotor and anti-torque rotor. Vestnik MAI, 16(5), 24-31.

Johnson, W. (2013). Rotorcraft aeromechanics. Cambridge university press. https://doi.org/10.1017/CBO9781139235655

Kelly, R., Jemcov, A., Rennie, M., Jumper, E. J., Whiteley, M., \& Goorskey, D. (2015). Computation of the aero-optical effect of a helicopter rotor wake using unsteady RANS and LES. Proceedings of 53rd AIAA Aerospace Sciences Meeting, 15. https://doi.org/10.2514/6.2015-0678

Kinzel, M. P., Cornelius, J. K., Schmitz, S., Palacios, J., Langelaan, J. W., Adams, D. S., \& Lorenz, R. D. (2019). An investigation of the behavior of a coaxial rotor in descent and ground effect. In AIAA Scitech 2019 Forum. https://doi.org/10.2514/6.2019-1098

Kritsky, B. S., Mahnyov, M. S., Mirgazov, R. M., Subbotina, P. N., \& Trebunskih, T. V. (2016). Aerodynamic characteristics calculation of single rotor blade using FLOWFD, ANSYS FLUENT and RC-VTOL. Civil Aviation High Technologies, 223, 77-83.

Leishman, J. G. (2006). Principles of helicopter aerodynamics. Cambridge university press.

Long, L., \& Fritz, T. E. (2004). Object-oriented unsteady vortex lattice method for flapping flight. Journal of Aircraft, 41(6), 1275-1290. https://doi.org/10.2514/1.7357

Potsdam, M., Smith, M., \& Renaud, T. (2009). Unsteady computations of rotor-fuselage interaction. Proceedings of the 35th European Rotorcraft Forum, 23.

Shcheglova, V. M. (2011). Aerodynamic calculation method of the main rotor taking into account diffusion of free vortices for small flight speeds. TsAGI Science Journal, 42, 179-203. https://doi.org/10.1615/TsAGISciJ.v42.i2.50

Shi, Y., Xu, Y., Xu, G., \& Wei, P. (2017). A coupling VWM/ $\mathrm{CFD} / \mathrm{CSD}$ method for rotor airload prediction. Chinese Jour- 
nal of Aeronautics, 30, 204-215. https://doi.org/10.1016/j. cja.2016.12.014

Singh, P., \& Friedmann, P. (2017). Application of vortex methods to coaxial rotor wake and load calculations in hover. Journal of Aircraft, 55, 1-9. https://doi.org/10.2514/6.2017-0051

Tan, J., Sun, Y., \& Barakos, G. (2018). Unsteady loads for coaxial rotors in forward flight computed using a vortex particle method. The Aeronautical Journal, 122, 1-22.

https://doi.org/10.1017/aer.2018.8

Wenping, S., Zhong-Hua, H., \& Qiao Zhi, D. (2007). Prediction of hovering rotor noise based on reynolds-averaged navierstokes simulation. Journal of Aircraft, 44, 1391-1395. https://doi.org/10.2514/1.28310

Zhao, Y., Shi, Y., \& Xu, G. (2017). Helicopter blade-vortex interaction airload and noise prediction using coupling CFD/ VWM method. Applied Sciences, 7(4), 381.

https://doi.org/10.3390/app7040381

\section{Notations}

Variables and functions

$\mathrm{c}$ - blade chord, $\mathrm{m}$;

$C_{\mathrm{n}}$ - blade section normal force coefficient, $(2 \cdot \mathrm{N}) /$ $\left(\rho \cdot(\omega R)^{2} \cdot c\right)$;

$C_{\mathrm{Q}}$ - rotor torque coefficient, $(2 \cdot \mathrm{Q}) /\left(\rho \cdot(\omega \mathrm{R})^{2} \cdot \pi \mathrm{R}^{3}\right)$;

$C_{\mathrm{T}}$ - rotor thrust coefficient, $(2 \cdot \mathrm{T}) /\left(\rho \cdot(\omega \mathrm{R})^{2} \cdot \pi \mathrm{R}^{2}\right)$;

$\mathrm{N}$ - normal force, $\mathrm{N}$;

$N_{\mathrm{b}}$ - number of blades;
$\mathrm{Q}$ - rotor torque, $\mathrm{N} \cdot \mathrm{m}$;

$\mathrm{R}$ - rotor radius, $\mathrm{m}$;

$\mathrm{T}$ - rotor thrust, $\mathrm{N}$;

$V_{\infty}$ - flight velocity, $\mathrm{m} / \mathrm{s}$;

$v_{y}$ - inductive velocity, $\mathrm{m} / \mathrm{s}$;

$\alpha_{R}$ - angle of rotor attack, degree;

$\mu$ - rotor advance ratio, $\mathrm{V} \cdot \cos \alpha_{\mathrm{R}} / \omega \mathrm{R}$;

$\theta$ - blade pitch angle, degree;

$\theta_{\mathrm{tw}}$ - blade twist, degree;

$\sigma$ - rotor solidity, $\mathrm{Nb} / \pi \mathrm{R}^{2}$;

$\omega \mathrm{R}$ - linear speed of the blade tips, $\mathrm{m} / \mathrm{s}$;

$\psi$ - blade azimuth angle, degree.

\section{Abbreviations}

BVI - phenomenon - vortices interact with the rotor blades; CFD - computational fluid dynamics;

CPU - central processing unit;

DES - Detached-Eddy Simulation;

FVM - free wake vortex models;

RANS - Reynolds-averaged Navier-Stokes equations;

URANS - Unsteady Reynolds-averaged Navier-Stokes equations;

VLM - vortex lattice method;

VPM - vortex particle method. 